\title{
EFEITO DE DIFERENTES TRATAMENTOS SUPERFICIAIS NA RESISTÊNCIA DE UNIÃO DE REPARO DE PORCELANA COM RESINA COMPOSTA
}

\section{EFFECT OF DIFFERENT SURFACE TREATMENTS ON THE BOND STRENGTH OF COMPOSITE RESIN AND PORCELAIN REPAIRS}

\author{
Hercules Jorge Almihatti ${ }^{1}$, Karin Hermana Neppelenbroek ${ }^{2}$, Elbio Holanda \\ Moura ${ }^{3}$, Vinícius Kleinübing Rhoden ${ }^{4}$, Nara Hellen Campanha ${ }^{2}$, Rosangela \\ Seiko $\mathrm{Seo}^{5}$ \\ 1 Autor para contato: Universidade Federal do Paraná - UFPR, Departamento de \\ Odontologia Restauradora, Curitiba, PR; (41) 33604038 e (41) 88023307; e-mail: \\ almilhatti@ufpr.br \\ 2 Universidade Estadual de Ponta Grossa - UEPG, Departamento de Odontologia. \\ 3 Universidade de Fortaleza - UNIFOR, Disciplina de Prótese. \\ 4 Centro Universitário de Maringá - CESUMAR \\ 5 Centro Universitário de Várzea Grande - UNIVAG, Disciplina de Prótese.
}

Recebido para publicação em 28/04/2008

Aceito para publicação em 02/11/2008

\begin{abstract}
RESUMO
Este estudo avaliou o efeito de diferentes tratamentos superficiais na resistência de união ao cisalhamento de uma cerâmica feldspática (Noritake EX-3), reparada com uma resina composta (Z-100). Quarenta corpos-de-prova da porcelana foram incluídos em resina acrílica, polidos (\# 320, \# 600) e divididos em 4 grupos $(\mathrm{n}=10)$ de acordo com os tratamentos superficiais: G1) sem tratamento (controle); G2) condicionamento com ácido fluorídrico a $10 \%$ por $60 \mathrm{~s}$; G3) asperização com ponta diamantada; G4) jateamento com Al2O3 (50 $\mu \mathrm{m})$. Após o tratamento, o silano (RelyX ceramic primer) e o adesivo (Scotchbond Multi-Purpose) foram aplicados nas superfícies da porcelana antes da união a $2 \mathrm{~mm}$ de resina composta. Após $24 \mathrm{~h}$ de armazenamento em água destilada a $37^{\circ} \mathrm{C}$ e $24 \mathrm{~h}$ de termociclagem $(1.000$ ciclos, $4^{\circ} \mathrm{C} / 60^{\circ} \mathrm{C}, 15 \mathrm{~s}$ cada), os corpos-de-prova foram submetidos ao teste de cisalhamento em máquina de ensaios universal, com velocidade de $0,5 \mathrm{~mm} / \mathrm{min}$. Os dados foram submetidos à análise de variância e ao teste de Tukey $(\alpha=0,05)$. Os tratamentos superficiais (G2=25,00 $\pm 2,99 \mathrm{MPa} ; \mathrm{G} 3=22,48 \pm 4,54 \mathrm{MPa}$; G4=24,18 $\pm 5,03 \mathrm{MPa}$ ) demonstraram significativamente $(\mathrm{P}<0,05)$ maiores valores de resistência de união quando comparados ao controle $(\mathrm{G} 1=11,57 \pm 2,06 \mathrm{MPa})$, mas não apresentaram diferenças significativas $(\mathrm{P}>0,05)$ entre si. A microscopia óptica revelou aproximadamente $80 \%$ de falhas adesivas para o grupo controle e $65 \%$ de falhas coesivas da porcelana para os grupos submetidos aos tratamentos superficiais. Foi possível concluir que os tratamentos superficiais avaliados melhoraram significativamente a resistência entre a cerâmica Noritake e a resina Z-100.
\end{abstract}


Palavras-Chave: Prótese parcial fixa. Resistência ao cisalhamento. Reparo. Resina composta. Porcelana dentária.

\begin{abstract}
This study evaluated the effect of different surface treatments on the shearing bond strength of a feldspathic porcelain (Noritake EX-3), repaired with a composite resin (Z-100). Forty porcelain specimens were mounted into acrylic resin, polished (\#320, \# 600) and divided into 4 groups ( $\mathrm{n}=10)$, according to the following surface treatments: G1) non-polished (control); G2) etched with $10 \%$ hydrofluoric acid gel for $60 \mathrm{~s}$; G3) ground with a high-speed diamond bur; G4) sandblasted with $50 \mu \mathrm{m}$ A2O3 with an intraoral sandblaster. After the treatment, the silane coupling agent (RelyX ceramic primer) and the adhesive (Scotchbond Multi-Purpose) were applied to the treated specimens, after being bonded with $2 \mathrm{~mm}$ of composite resin. After a 24-hour storage in distilled water at $37 \mathrm{oC}$, and an additional 24-hour thermocycling period $(1,000$ cyles, $4 \mathrm{oC} / 60 \mathrm{oC}$, dwell time: $15 \mathrm{~s})$, the specimens were subjected to a shearing force in a universal testing machine at a crosshead speed of $0.5 \mathrm{~mm} /$ min. Data were analyzed by ANOVA, followed by the Tukey test $(\alpha=0.05)$. All surface treatments $(\mathrm{G} 2=25.00 \pm 2.99 \mathrm{MPa} ; \mathrm{G} 3=22.4 \pm 4.54 \mathrm{MPa} ; \mathrm{G} 4=24.18 \pm 5.03$ $\mathrm{MPa})$ showed significantly higher bond strength $(\mathrm{P}<.05)$ when compared with the control group ( $\mathrm{G} 1=11.57 \pm 2.06 \mathrm{MPa})$, but they did not present significant differences $(\mathrm{P}>.05)$ among one another. Optical microscopy showed $80 \%$ adhesive failure for control specimens, and $65 \%$ of cohesive failure in the porcelain on treated specimens. These results evinced that intraoral repairs of Noritake porcelain bonded with Z-100 composite resin can significantly improve surface treatments.
\end{abstract}

Keywords: Partial fixed denture. Shearing strength. Repair. Composite resin. Porcelain.

\section{Introdução}

O desenvolvimento de modernos sistemas cerâmicos e adesivos tornou possível restaurar elementos dentários danificados e, dentro de limitações relativas à resistência mecânicas, até mesmo substituí-los proteticamente, sem a necessidade do uso de metais. Porém, reabilitações orais extensas ainda são dependentes do reforço propiciado por infra-estruturas metálicas e, nesse contexto, as próteses metalocerâmicas ainda constituem uma das principais opções (CHUNG; HWANG, 1997).

Considerando as limitações das próteses estéticas de porcelana e a complexidade da cavidade bucal na qual estão expostas, não é incomum a ocorrência de fraturas no revestimento estético, sendo freqüentemente observada exposição do substrato metálico. De acordo com Özcan (2003a, 2003b), alguns estudos clínicos indicam uma prevalência de $5 \%$ a $10 \%$ de falhas após 10 anos de uso e tal ocorrência gera um desconforto estético, funcional e econômico, tanto para o profissional como para o paciente.

Infelizmente, devido ao processo de confecção, o reparo intra-oral utilizando porcelana torna-se impossível e a substituição das próteses pode não ser viável devido a fatores como custo elevado, necessidade de novo desgaste da estrutura dental, falta de disponibilidade do paciente e dificuldade de remoção das próteses (GREGORY; MOSS, 1990). Assim, reparos utilizando materiais compósitos, associados a sistemas adesivos, podem ser utilizados objetivando restabelecer a função e a estética de restaurações que perderam parte de sua estrutura. (APPERLDOORN et al., 1993).

Dessa forma, o sucesso clínico torna-se de- 
pendente da integridade e estabilidade da união entre porcelana, metal e resina composta, alcançado graças à união química e mecânica desses materiais (ÖZCAN, 2003b). Diferentes técnicas e métodos têm sido empregados com o objetivo de otimizar a união da superfície cerâmica fraturada com o sistema adesivo e resina composta: condicionamento com ácido fluorídrico, fosfórico ou flúor fosfato acidulado (SARAÇOGLU et al., 2004; ROBBINS, 1997; LEIBROCK et al., 1999), jateamento com partículas de óxido de alumínio- $\mathrm{Al}_{2} \mathrm{O}_{3}$ (WOLF et al., 1992), asperização com pontas diamantadas (KUPIEC et al.,1996) e/ou aplicação de um agente de silano (AGRA et al., 1993; DELLA BONA; NORTHEAST, 1994) sobre a cerâmica fraturada.

Dessa forma, considerando as inúmeras possibilidades de tratamentos superficiais para reparos em próteses cerâmicas, o presente trabalho teve como objetivo avaliar a resistência de união ao cisalhamento entre uma porcelana feldspática, após diferentes tratamentos superficiais, e uma resina composta.

\section{Material e método}

Inicialmente foram confeccionadas 40 pastilhas de porcelana feldspática (quadro 1) por meio de aglutinação do pó e água destilada em uma matriz circular de aço inoxidável, com uma perfuração central com as seguintes dimensões: 8,0 $\mathrm{mm}$ de diâmetro e 3,0 $\mathrm{mm}$ de espessura. Com o auxílio de um pincel macio, pequenas porções da mistura pó/líquido foram sendo compactadas no interior da matriz, sobre uma placa de vidro, até seu completo preenchimento. Em seguida, a pastilha foi removida com um sacador e levada ao forno (Vulcano, EDG Equipamentos e Controle, São Carlos, SP, Brasil) para sinterização, com temperaturas iniciando em $600^{\circ} \mathrm{C}$ até $930^{\circ} \mathrm{C}$ sob vácuo e mais 1 minuto a $935^{\circ} \mathrm{C}$ sem vácuo, conforme instrução do fabricante. Após a sinterização e resfriamento à temperatura ambiente, todas as pastilhas de porcelana foram incluídas individualmente em tubos de PVC (25,4 $\mathrm{mm}$ de diâmetro e $20,0 \mathrm{~mm}$ de altura), com resina acrílica incolor autopolimerizável, deixando uma das superfícies da pastilha exposta e nivelada na parte superior do tubo de PVC.

Em seguida, a superfície da porcelana exposta no tubo de PVC foi polida (Metaserv 2000, Buehler, Brasil) com lixas seqüenciais (\# 320, \# 600) de carbeto de silício (Norton, Saint-Gobain Abrasivos Ltda., Brasil) até se obter uma superfície uniforme e livre de irregularidades. Em seguida, as superfícies polidas foram lavadas com escova dental e detergente e submetidas à limpeza final com vapor d'água em aparelho vapor Jet I (EDG Equipamentos e Controles Ltda., São Carlos, SP, Brasil). O mesmo procedimento foi executado em todos os tubos de PVC, os quais foram então divididos aleatoriamente em quatro grupos iguais $(\mathrm{n}=10)$ de acordo com os seguintes grupos experimentais e materiais utilizados (quadro 1):

Grupo G1 (Controle): Aplicação do adesivo e da resina composta de acordo com as instruções do fabricante.

Grupo G2 (Ácido fluorídrico): Condicionamento com o ácido fluorídrico a $10 \%$ por $60 \mathrm{seg}$., lavagem com água corrente e secagem com jato de ar, aplicação do silano, do adesivo e da resina composta de acordo com as instruções do fabricante.

Grupo G3 (Ponta diamantada): Asperização com uma ponta diamantada (n. 4137, KG Sorensen, Brasil) acoplada a uma caneta de alta rotação sem refrigeração por $10 \mathrm{seg}$., lavagem com água corrente e secagem com jato de ar, aplicação do silano, do adesivo e da resina composta de acordo com as instruções do fabricante.

\begin{tabular}{|l|l|}
\hline \multicolumn{1}{|c|}{ Material/ Marca Comercial } & \multicolumn{1}{c|}{ Fabricante } \\
\hline Porcelana feldspática Noritake EX- 3 & Noritake, Japão \\
\hline Ácido fluorídrico $10 \%$ & Dentsply, Brasil \\
\hline $\begin{array}{l}\mathrm{Al}_{2} \mathrm{O}_{3} \text { de } 50 \mu \mathrm{m}-\text { Cobra }\left(99,5 \% \mathrm{Al}_{2} \mathrm{O}_{3} \text { e máx }\right. \\
\left.\text { de } 0,06 \% \mathrm{SiO}_{2}\right)\end{array}$ & Renfert GmbH, Alemanha \\
\hline Silano - Ceramic Primer ( Rely X) & 3M ESPE, USA \\
\hline Sistema adesivo - Scotchbond Multi- Purpose & 3M ESPE, USA \\
\hline Resina Composta - Restaurador Z-100 & 3M ESPE, USA \\
\hline Ponta Diamantada $n^{\circ} 4137$ & KG Sorensen, Brasil \\
\hline
\end{tabular}

Quadro 1- Materiais utilizados 
Grupo G4 (Jateamento com $\mathrm{Al}_{2} \mathrm{O}_{3}$ ): Jateamento com partículas de $\mathrm{Al}_{2} \mathrm{O}_{3}$ de $50 \mu \mathrm{m}$ (Microjato, Bio Art, São Carlos, SP) por $10 \mathrm{~s}$, lavagem com água corrente e secagem com jato de ar, aplicação do silano, do adesivo e da resina composta de acordo com as instruções do fabricante.

O adesivo foi aplicado com pontas descartáveis e polimerizado de acordo com as instruções do fabricante com aparelho fotopolimerizador (Curing Ligth XL 3000, 3M, Alemanha), com intensidade de luz de aproximadamente $480 \mathrm{~mW} / \mathrm{cm}^{2}$, aferida por radiômetro analógico (Gnatus, Ribeirão Preto, Brasil) para cada corpo-de-prova.

A resina composta foi aplicada com espátula de inserção em duas camadas ( $1 \mathrm{~mm}$ de espessura cada uma) polimerizada com o mesmo aparelho citado, porém com o auxílio de um matriz metálica bipartida com diâmetro interno de $4,0 \mathrm{~mm}$ e 2,0 $\mathrm{mm}$ de espessura. Essa matriz foi posicionada sobre a superfície da amostra e estabilizada no tubo de PVC por um anel metálico centralizador (ALMEIDA et al., 2006) para permitir a aplicação e a polimerização da resina de acordo com as instruções do fabricante, aparelho e intensidade de luz como descrito anteriormente (figura 1).

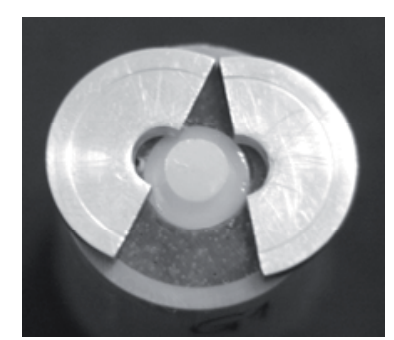

Figura 1 - Anel centralizador removido e matriz bipartida aberta para liberação do corpo-de-prova.

Finalizada a confecção dos corpos-de-prova, todos os grupos foram armazenados em água destilada em estufa de cultura regulada à temperatura constante de $37^{\circ} \mathrm{C}$ por 24 horas. Após esse período, os corpos-de-prova foram submetidos a 1.000 ciclos térmicos em banhos alternados em água quente $\left(60^{\circ} \mathrm{C}\right)$ e fria $\left(4^{\circ} \mathrm{C}\right)$, com permanência de $15 \mathrm{seg}$. em cada temperatura. $\mathrm{O}$ equipamento utilizado foi uma máquina de simulação de ciclos térmicos modelo MSCT-3 (fabricante, São Carlos, SP, Brasil). Em seguida, os corpos-de-prova foram submetidos aos ensaios mecânicos de cisalhamento em uma máquina de ensaios EMIC (Modelo DL 2000, Equipamentos e Sistemas de Ensaios Ltda., Brasil) com o auxílio de um dispositivo especialmente construído e velocidade de carregamento de 0,5 $\mathrm{mm} / \mathrm{min}$. Para o cálculo da tensão de cisalhamento $(\sigma)$, foi utilizada a seguinte expressão: $\sigma(\mathrm{MPa})=$ $\mathrm{F}_{\text {máx }}(\mathrm{N}) / \mathrm{A}_{\text {união }}\left(\mathrm{mm}^{2}\right)$, onde $\mathrm{F}_{\text {máx }}$ representa a forca máxima de rompimento fornecida pelo computador da máquina de ensaios e $\mathrm{A}_{\text {união }}$ a área de união entre a porcelana e a resina composta (figura 2).

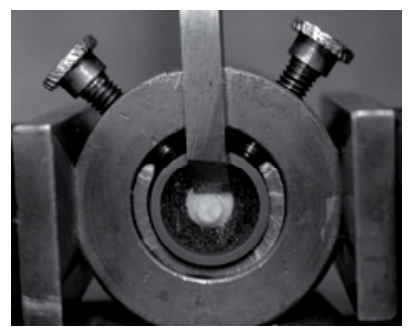

Figura 2 - Corpo-de-prova posicionado para realização do ensaio mecânico de cisalhamento.

Após os testes de cisalhamento, a face de união fraturada foi observada em uma lupa estereoscópica (Carlzeiss-Jenna, Alemanha) capaz de aumentar 40 vezes o objeto, sendo as falhas classificadas em adesiva, coesiva ou mista.

Para avaliar o efeito dos diferentes tratamentos superficiais, os valores das tensões de cisalhamento foram submetidos à análise de variância complementada pelo teste de comparações múltiplas de Tukey, ambos ao nível de 5\% de significância.

\section{Resultado}

Os valores médios das tensões de cisalhamento e seus respectivos intervalos de $95 \%$ de confiança estão representados na Figura 3. De acordo com a análise estatística dos dados, foi possível observar que os Grupos G2=25,0 Mpa, G3=22,5 Mpa e $\mathrm{G} 4=24,2 \mathrm{MPa}$ apresentaram maiores valores de resistência de união $(\mathrm{p}<0,05)$ quando comparados ao Grupo G1=11,6 MPa (controle). Porém, os tratamentos avaliados (condicionamento ácido, asperização com ponta diamantada e jateamento com $\mathrm{Al}_{2} \mathrm{O}_{3}$ ) não apresentaram diferenças estatisticamente significativas $(p>0,05)$ entre si. A microscopia 


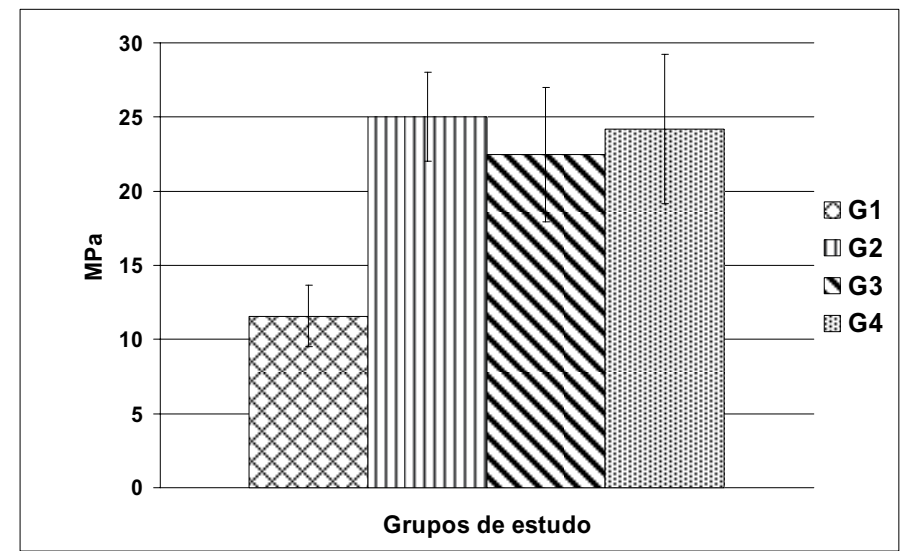

Figura 3 - Representação gráfica das médias de resistência adesiva, em MPa, de acordo com os tratamentos superficiais. As barras verticais indicam um intervalo de $95 \%$ de confiança para a média populacional.

óptica revelou aproximadamente $80 \%$ de falhas adesivas para o grupo controle e $65 \%$ de falhas coesivas da porcelana para os grupos submetidos aos tratamentos superficiais.

\section{Discussão}

Embora as restaurações metalocerâmicas desempenhem importante função em reabilitações orais por associarem a excelente estética e biocompatibilidade da cerâmica com a resistência mecânica das estruturas metálicas, estudos clínicos indicam que a prevalência de falhas e fraturas da cerâmica pode variar entre $5 \%$ a $10 \%$ após 10 anos de uso (OZCAN, 2003a). De acordo com Ozcan (2003b), as fraturas podem ocorrer de três modos: fratura somente da porcelana, fratura com exposição de porcelana e metal e fratura com exposição de grande superfície de metal. Dessa forma, o profissional deve estar capacitado para identificar o tipo de fratura e quais os melhores procedimentos a serem realizados, considerando suas limitações e observando características importantes das restaurações como integridade marginal, adaptação da estrutura metálica, oclusão e preservação dos tecidos de suporte (PAMEIJER et al., 1996; ROBBINS, 1998). De acordo com Galiatsatos (2005), os métodos de reparo podem ser classificados em diretos ou indiretos. Reparos indiretos envolvem a confecção de restaurações laboratoriais que devem ser cimentadas sobre a estrutura fraturada. Reparos diretos são mais comumente utilizados e se fundamentam na aplicação direta de camadas de resina composta associada a sistemas adesivos e diferentes tratamentos da superfície cerâmica fraturada. Neste estudo, o reparo foi realizado pela técnica direta através da aplicação de resina composta associada ao sistema adesivo em substrato cerâmico previamente tratado.

Dentre os métodos capazes de produzir alterações superficiais na cerâmica, a literatura relata a utilização de asperização com pontas diamantadas (LACY et al., 1988; SULIMAN et al., 1993; EIKENBERG; SHURTLEFF, 1996; COCHRAN et al., 1998; KUMBULOGLU et al., 2003), jateamento com óxido de alumínio (BERTOLOTTI et al, 1989; WOLF et al., 1992; BERKSUN; SAGLAM, 1994; CHUNG; HWANG, 1997; ROBBINS, 1997), aplicação de agentes condicionadores, como o flúor fosfato acidulado e o ácido fluorídrico (SENDA et al., 1989; WOLF et al., 1993; TYLKA; STEWART, 1994; HOLLWEG, 1997; DELLA BONA; VAN NOORT, 1998, KUKIATTRAKOON; THAMMASITBOON, 2007). A literatura também relata a utilização de métodos tais como jateamento com partículas de óxido de alumínio- $\mathrm{Al}_{2} \mathrm{O}_{3}$ (SHAHVERDI et al., 1998; WOLF et al., 1992; KIM et al., 2005; YESIL et al., 2007), asperização com pontas diamantadas (Kupiec et al., 1996), aplicação de silano (AGRA et al., 1993, DELLA BONA; NORTHEAST, 1994) e deposição de uma camada de sílica (SiOx) (OZCAN et al., 2006) sobre a cerâmica fraturada.

Nesta pesquisa, os tratamentos modificadores da superfície cerâmica selecionados foram: asperização com ponta diamantada, condicionamento com ácido fluorídrico, jateamento com partículas de óxido de alumínio, para obtenção de retenções mecânicas e, aplicação de silano, para obtenção de adesão química. Os resultados deste estudo mostraram que os tratamentos da superfície cerâmica foram efetivos quando comparados com o grupo controle, pois enquanto este apresentou uma resistência de 11,6 MPa, os grupos que receberam os tratamentos apresentaram valores estatisticamente superiores e iguais entre si: condicionamento com 
ácido fluorídrico $=25,0 \mathrm{MPa}$; asperização com ponta diamantada $=22,5 \mathrm{MPa}$ e jateamento com partículas de óxido de alumínio = 24,2 MPa. Pode-se, portanto, concluir que todos os tratamentos foram efetivos e que podem ser utilizados para realizar os reparos, desde que associados com um agente químico de união silano. Para Matinlinna et al. (2004), a adesão efetiva entre cerâmica e resina depende de um adequado agente intermediário de ligação entre esses diferentes materiais: o silano, molécula bifuncional com uma parte organofuncional que se polimeriza com a matriz orgânica (resina), e a outra parte silicofuncional que se liga com o substrato inorgânico (cerâmica). Em ambos os casos, por ligações covalentes.

Os resultados desta pesquisa estão de acordo com os autores (CHUNG; HWANG, 1997; JARDEL, 1999) que concluíram em seus estudos que o tratamento de superfície com ácido fluorídrico aumenta a resistência de união entre a resina composta e a porcelana. Embora alguns autores (LEIBROCK et al., 1999; CANAY et al., 2001; KUSSANO et al., 2003) questionem o seu efeito danoso quando em contato com tecidos moles, outros autores, como Van Noort (1994) afirmam que o seu uso, de forma controlada, não causa nenhum dano. A efetividade da utilização de pontas diamantadas também está de acordo com o estudo de Saygili e Sahmali (2003). Mesmo sendo questionado por promover trincas no corpo da porcelana (Ozcan, 2003b), esse método tem sido empregado em razão de sua praticidade e pelo fato de dispensar a aquisição de materiais e instrumentais adicionais (EIKENBERG; SHURTLEFF, 1996; COCHRAN et al., 1998; KUMBULOGLU et al., 2003). Apesar de exigir o emprego de instrumento (microjateador) e material específico $\left(\mathrm{Al}_{2} \mathrm{O}_{3}\right)$ muitas vezes não disponíveis nos consultórios odontológicos, o jateamento com partículas abrasivas de óxido de alumínio também se mostrou efetivo neste estudo, confirmando a indicação sugerida por alguns estudos (BERTOLOTTI et al., 1989; WOLF et al., 1992; BERKSUN; SAGLAM, 1994; CHUNG; HWANG, 1997; ROBBINS, 1997).

Diante dos resultados obtidos nesta pesquisa, pode-se concluir que as técnicas propostas melhoraram a resistência ao cisalhamento entre resina composta e porcelana. Essa conclusão está de acordo com Phoenix e Shen (1995) que afirmam que o tratamento mecânico e químico das superfícies de porcelana feldspática produzem um aumento total das áreas de superfície e aumento total da energia de superfície e essa mudança de topografia de superfície é responsável pelo aumento da resistência de união. Porém, novas pesquisas devem ser conduzidas e padronizadas para que possam ser utilizadas de forma mais segura e efetiva na indicação de materiais e métodos para a realização de reparos em cerâmicas.

\section{Conclusão}

De acordo com a metodologia empregada e os resultados obtidos neste estudo, pode-se concluir que, quando comparados aos obtidos com o grupo controle (sem tratamento superficial), os valores de resistência de união entre a cerâmica Noritake e a resina Z-100 foram melhorados com os três tratamentos superficiais testados (asperização com ponta diamantada, condicionamento com ácido fluorídrico e jateamento com partículas de óxido de alumínio).

\section{REFERÊNCIAS}

AGRA, C.M., GARÓFALO, J.C., VIEIRA, G.F. Silano: análise da importância deste material na união química entre porcelana e resina composta. Âmbito Odontol, v.3, p. 32631, 1993.

ALMEIDA, J.G.S.P. et al.. Shear bond strength of metalceramic repair systems. J Prosthet Dent, v. 96, n. 3, p. 165 173, 2006.

APPELDOORN, R.E., WILWEDING, T.M., BARKMEIER, W.W. Bond strength of composite resin to porcelain with newer generation porcelain repair systems. J Prosthet Dent, v. 70, n. 1, p. 6-11, 1993.

BERKSUN, S.; SAGLAM, S. Shear strength of composite bonded porcelain-to-porcelain in a new repair system. J Prosthet Dent, v.71, n. 4, p. 423-8, 1994.

BERTOLOTTI, R.L.; LACY, A.M,, WATANABE, L.G. Adhesive monomers for porcelain repair. Int J Prosthodont, v. 2, n. 5, p. 483-9, 1989.

CANAY, S.; HERSEK, N.; ERTAN, A. Effect of different acid 
treatments on a porcelain surface. J Oral Rehabil, v. 28, n. 1, p. 95-101, 2001

CHUNG, K.H.; HWANG, Y.C. Bonding strengths of porcelain repair systems with various surface treatments. J Prosthet Dent, v. 78, n. 3, p. 267-74, 1997.

COCHRAN, M.A. et al. Tensile bond strengths of five porcelain repair systems. Oper Dent, v. 13, n. 4, p. 162-7, 1998.

DELLA BONA, A.; NORTHEAST, S.E. Shear bond strength of resin bonded ceramic after different try-in procedures. $\mathbf{J}$ Dent, v. 22, n. 2, p. 103-7, 1994.

; VAN NOORT, R. Ceramic surface preparations for resin bonding. Am J Dent, v. 11, n. 6, p. 276-80, 1998.

EIKENBERG, S.; SHURTLEFF, J. Effect of hydration on bond strength of a silane-bonded composite to porcelain after seven months. Gen Dent, v. 44, n. 1, p. 58-61, 1996.

GALIATSATOS, A.A. An indirect repair techinique for fractured metal-ceramic restorations: A clinical report. J Prosthet Dent, v. 93, n. 4, p. 321-3, 2005.

GREGORY, W.A.; MOSS, S.M. Effects of heterogeneous layers of composite and time on composite repair of porcelain. Oper Dent, v. 15, n. 1, p. 18-22, 1990.

HOLLWEG, H. Avaliação da resistência de união entre cerâmica / resina composta através de ensaios de cisalhamento em função de diferentes tipos de cerâmicas. Bauru, 1997. 103p. Dissertação (Mestrado em) - Faculdade de Odontologia de Bauru, Universidade de São Paulo.

JARDEL, V. Correlation of topography to bond strength of etched ceramic. Int J Prosthodont, v. 12, n. 1, p. 59-64, 1999.

KIM, B.K. et al. The influence of ceramic surface treatments on the tensile bond strength of composite resin to all-ceramic coping materials. J Prosthet Dent, v. 94, n. 4, p. 357-62, 2005.

KUKIATTRAKOON, B.; THAMMASITBOON, K. The effect of different etching times of acidulated phosphate fluoride gel on the shear bond strength of high-leucite ceramics bonded to composite resin. J Prosthet Dent, v. 98, n. 1, p. 17-23, 2007.

KUMBULOGLU, O. et al.. Intra-oral adhesive systems for ceramic repairs: a comparasion. Acta Odontol Scand, v. 61, n. 5, p. 268-72, 2003.

KUPIEC, K.A. et al. Evaluation of porcelain surface treatments and agents for composite-to-porcelain repair. J Prosthet Dent, v. 76, n. 2, p. 119-24, 1996.

KUSSANO, C.M. et al. Evaluation of shear bond strength of composite to porcelain according to surface treatment. Braz Dent J, v. 14, n. 2, p. 132-5, 2003.

LACY, A.M. et al. Effect of porcelain surface treatment on the bond to composite. J Prosthet Dent, v. 60, n. 3, p. 28891, 1988.

LEIBROCK, A. et al. In vitro study of the effect of thermoand load-cycling on the bond strength of porcelain repair systems. J Oral Rehabil, v. 26, n. 2, p. 130-7, 1999.

MATINLINNA J.P., et al. An introduction to silanes and their clinical applications in dentistry. Int J Prosthodont, v. 17, n. 2, p. 155-64, 2004.

OZCAN, M., et al. Comparison of repair methods for ceramicfused-to-metal crowns. J Prosthodont, v. 15, n. 5, p.283-8, 2006.

. Evaluation of alternative intra-oral repair techniques for fractured ceramic-fused-to-metal restorations. J Oral Rehabil, v. 30, n. 2, p. 194-203, 2003 b.

. Fracture reasons in ceramic-fused-to-metal restora-

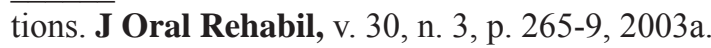

PAMEIJER, C.H.; LOUW, N.P.; FISCHER, D. Repairing fractured porcelain: how surface preparation affects shear force resistance. J Am Dent Assoc, v. 127, n. 2, p. 203-9, 1996.

PHOENIX, R.D.; SHEN, C. Characterization of treated porcelain surfaces via dynamic contact angle analysis. Int $\mathbf{J}$ Prosthdont, v. 8, n. 2, p. 187-94, 1995.

ROBBINS, J.W.. Intraoral repair of the fractured porcelain restoration. Oper Dent, v. 23, p. 203-7, 1997.

SARAÇOGLU, A.; CURA, C.; ÇOTERT, H.S. Effect of various surface treatment methods on the bond strength of the heat-pressed ceramic samples. J Oral Rehabil, v. 31, n. 8, p. 790-7, 2004.

SAYGILI, G.; SAHMALI, S. Effect of ceramic surface treatment methods on the bond strength on the heat-pressed ceramic samples. J Oral Rehabil, v. 31, n. 8, p. 790-7, 2003.

SENDA, A.; SUZUKY, M.; JORDAN, R.E. The effect of fluorides and hydrofluoric acids on porcelain surfaces. J Dent Res, v. 68, p. 236, 1989.

SHAHVERDI, S.; CANAY, Ş.,; ŞAHIN, E. et al. Effects of different surface treatment methods on the bond strength of composite resin to porcelain. J Oral Rehabil, v. 25, n. 9, p. 699-705, 1998.

SULIMAN, A.A.; SWIGT, E.J.; PERDIGAO, J. Effects of surface treatment and bonding agents on bond strength of composite resin to porcelain. J Prosthet Dent, v. 70, n. 2, p. 118-20, 1993.

TYLKA, D.F.; STEWART,G.P. Comparison of acidulated phosphate fluoride gel and hydrofluoric acid etchants for porcelain-composite repair. J Prosthet Dent, v. 72, n. 2, p. 121-7, 1994.

VAN NOORT, R. Introduction to dental material. In: VAN NOORT R. Principles of adhesion. St. Luis: Mosby; 1994. 
cap. 9, p. 61- 71 .

WOLF, D.M., POWERS, J.M., O'KEEFE, K.L. Bond strength of composite to etched and sandblasted porcelain. Am J Dent, v. 6 , n. 3, p. 155-8, 1993.

Bond strength of composite to porcelain treated with new porcelain repair agents. Dent Mater, v. 8, n. 3, p. 158-61, 1992.

YESIL, Z.D. et al. Effect of different surfaces and surface applications on bonding strength of porcelain repair material. N Y State Dent J, v. 73, n. 3, p. 28-32, 2007. 